\title{
Singularities with exact Poincare complex but not quasihomogeneous
}

\author{
Gerhard PFISTER and HaNS SCHÖNEMANN
}

ABSTRACT. We give examples of complete intersections in $C^{3}$ with exact Poincarè complex but not quasihomogeneous using the classification of C.T.C. Wall and the algorithm of Mora.

\section{INTRODUCTION}

$1971 \mathrm{~K}$. Saito gave a nice numerical characterization for a hypersurface singularity to admit a $C^{*}$-action $\left((X, 0)\right.$ admits a good $C^{*}$-action (also called quasihomogeneous) if $\bar{O}_{x, 0} \rightarrow C\left[\left[x_{1}, \ldots x_{\mathrm{m}}\right]\right] / I$ and $I=\left(p_{1}, \ldots p_{n}\right)$ is an ideal generated by quasihomogeneous polynomials of positive degree $\mathrm{d}_{i}$ with respect to the weights $w_{1}, \ldots w_{m},\left(w_{i}>0, w_{i} \in Z\right)$, i.e. $\left.p_{i}\left(\lambda^{w_{1}} x_{1}, \ldots \lambda^{w_{m}} x_{m}\right)=\lambda_{i} d_{i}\right)$ (cf. [8]):

Let $(X, 0) \subseteq\left(C^{m}, 0\right)$ be the germ of an isolated hypersurface singularity and denote by $\mu(\overline{X, 0})$ the Milnor number of $(X, 0)$ - a topological invariant of the singularity - and by $\tau(X, 0)$ the Tjurina number of $(X, 0)$ - the dimension of the mini-versal deformation of $(X, 0)$, an analytical invariant - then the following conditions are equivalent:

(1) $(X, 0)$ admits a good $C^{*}$-action

(2) $\mu(X, 0)=\tau(X, 0)$

(3) the Poincarè complex of $(X, 0)$

$$
0 \rightarrow C \rightarrow O_{x, 0} \rightarrow \Omega_{x, 0}^{1} \rightarrow \Omega_{x, 0}^{2} \rightarrow \ldots \rightarrow \Omega_{x, 0}^{m} \rightarrow 0
$$

is exact.

1980 Mathematics Subject Classification (1985 revision): 14B05.

Editorial de la Universidad Complutense. Madrid, 1989. 
The invarants $\mu$ and $\tau$ can be computed as the dimension of certain Artinian rings:

If $(X, 0)$ is defined by $f=0, f \in C\left\{x_{1}, \ldots, x_{m}\right\}$ a convergent power series then

$$
\begin{gathered}
\mu(X, 0)=\operatorname{dim}_{C} C\left\{x_{1}, \ldots, x_{m}\right\} /\left(\frac{\partial f}{\partial x_{1}}, \ldots, \frac{\partial f}{\partial x_{m}}\right) \\
\tau(X, 0)=\operatorname{dim}_{C} C\left\{x_{1}, \ldots, x_{m}\right\} /\left(f, \frac{\partial f}{\partial x_{1}}, \ldots, \frac{\partial f}{\partial x_{m}}\right)
\end{gathered}
$$

A similar numerical characterization of quasihomogeneous singularities (not being hypersurfaces) can be given for curve singularities (cf. [3], [10]). Especially if $(X, 0) \subseteq\left(C^{m}, 0\right)$ is a complete intersection curve with isolated singularity, then the following conditions are equivalent:

(1) $(X, 0)$ is quasihomogeneous, i.e. admits a good $C^{*}$-action

(2) $\mu(X, 0)=\tau(X, 0)$

If $(X, 0)$ is quasihomogeneous then the Poincarè complex

$$
0 \rightarrow C \rightarrow O_{x, 0} \rightarrow \Omega_{X, 0}^{\prime} \rightarrow \Omega_{x, 0}^{2} \rightarrow \ldots \rightarrow \Omega_{X, 0}^{m} \rightarrow 0
$$

is exact. (2).

It is natural to ask whether Saitos result can be generalized i.e. (3) implies

It is the purpose of this note to show that this is not the case. We give examples of complete intersections in $C^{3}$ with exact Poincaré complex but not quasihomogeneous.

The idea to construct these examples is as follows:

C.T.C. Wall gave a classification of the unimodal complete intersection singularities, especially he computed the Milnor number

$$
\mu(X, 0)=\operatorname{dim} \Omega_{X, 0}^{1} / d O_{x .0}(\text { cf. [11]). }
$$

If $f, g \in C\{x, y, z\}$ are power series defining an isolated curve singularity $(X, 0)$, then

$$
\tau(X, 0)=\operatorname{dim}_{C} C\{x, y, z,\} /\left(f, g, M_{1}, M_{2}, M_{\mathrm{J}}\right)
$$


(cf. [4]), $M_{i}$ the 2-minors of the Jacobian matrix of $f, g$.

Using Mora's algorithm to compute a Gröbner base (cf. [6]) one can compute $\tau(X, 0)$ and decide whether $(X, 0)$ is quasihomogeneous or not.

To prove the exactness of the Poincaré complex

$$
0 \rightarrow C \rightarrow O_{x, 0} \rightarrow \Omega_{X, 0}^{1} \rightarrow \Omega_{X, 0}^{2} \rightarrow \Omega_{X, 0}^{3} \rightarrow 0
$$

we use a result of Reiffen (cf. [7]):

$$
\Omega_{X, 0}^{1} \stackrel{d_{2}}{\rightarrow} \Omega_{X, 0}^{2} \stackrel{d_{3}}{\rightarrow} \Omega_{X, 0}^{3} \rightarrow 0
$$

is exact iff

$$
(f, g) \Omega_{c^{3}, 0} \subseteq d\left((f, g) \Omega_{c_{0}{ }_{0}}\right)
$$

This condition is always satisfied if $f$ and $g$ are quasihomogeneous polynomials (not necessarily with the same weights).

It remains to check that

$\operatorname{ker} d_{2}=d_{1} O_{x, 0}$, i.e.

$$
\begin{aligned}
\mu(X, 0)=\operatorname{dim} \Omega_{x, 0}^{1} / d_{1} O_{x, 0} & =\operatorname{dim} \Omega_{x, 0}^{1} / \operatorname{ker} d_{2} \\
& =\operatorname{dim} d_{2} \Omega_{x, 0}^{1} \\
& =\operatorname{dim} \operatorname{ker} d_{3} \\
& =\operatorname{dim} \Omega_{x, 0}^{2}-\operatorname{dim} \Omega_{x, 0}^{3}
\end{aligned}
$$

Now $\operatorname{dim} \Omega_{x, \theta}^{3}=C\{x, y, z\} /\left(\frac{\partial f}{\partial x}, \frac{\partial f}{\partial y}, \frac{\partial f}{\partial z}, \frac{\partial g}{\partial x}, \frac{\partial g}{\partial y}, \frac{\overline{\partial g}}{\partial z}\right)$ and $\operatorname{dim} \Omega_{x, 0}^{2}=C\{x, y, z\} / M$,

\section{$M$ generated by}

$(f, 0,0),(0, f, 0),(0,0, f),(g, 0,0),(0, g, 0),(0,0, g)$

$$
\begin{aligned}
& \left(\frac{\partial f}{\partial y}, \frac{\partial f}{\partial z}, 0\right),\left(\frac{\partial f}{\partial x}, 0,-\frac{\partial f}{\partial z}\right),\left(0, \frac{\partial f}{\partial x}, \frac{\partial f}{\partial y}\right),\left(\frac{\partial g}{\partial y}, \frac{\partial g}{\partial z}, 0\right), \\
& \left(\frac{\partial g}{\partial x}, 0,-\frac{\partial g}{\partial z}\right),\left(0, \frac{\partial g}{\partial x}, \frac{\partial g}{\partial y}\right) .
\end{aligned}
$$


Using again Mora's algorithm we can compute $\operatorname{dim} \Omega_{x, 0}^{3}$ and $\operatorname{dim} \Omega_{x, 0}^{2}$ and decide whether the Poincarè complex is exact or not. We were guided by a computer giving us the Gröbner bases and the corresponding dimensions for many examples. Especially we will give the invariants $\tau(X, 0), \operatorname{dim} \Omega_{x, 0}^{2}$, $\operatorname{dim} \Omega_{x, 0}^{3}$ for all singularities in C.T.C. Wallss classification ( $(3)$.

Also as an application we compare in $\S 4$ Walls classification of quasihomogeneous singularities with the corresponding classification of Aleksandrov (cf. [1]).

In $\S 1$ we will give the idea of Mora's algorithm to be able to follow our computations.

In $\S 2$ we give the examples mentioned above.

\section{MORA'S ALGORITHM}

We will describe the algorithm to compute a Gröbner base of a submodule of a free module. This algorithm is a modification of Buchberger's algorithm to the case of modules over local rings (cf. [2], [6]). The actual implementation by the authors on Atari, IBM-compatible PC and MacIntosh is much more sophisticated (using some extra results and ideas to shorten the computations) as the idea described here.

Let $R=C\left\{x_{1}, \ldots, x_{m}\right\}$ and $x^{g}=x_{i}^{a} \ldots x_{m}^{a_{m}}, x^{\underline{b}}=x_{1}^{b_{1}} \ldots x_{m}^{b_{m}}$ be monomials in $R$.

Definition 1: $x^{a}<x^{\underline{b}}$ if $\underline{a} \neq \underline{b}$ and either $\sum a_{i}<\sum b_{i}$ or $\sum a_{i}=\sum b_{i}$ and $a_{1}=$ $b_{1}, \ldots, a_{r}=b_{r} a_{r+1}<b_{r+1}$. We will write $x^{a} \leq x^{\underline{b}}$ if $x^{a}<x^{b}$ or $x^{\underline{a}}=x^{\underline{b}}$.

Definition 2: Let $x_{j}^{q}=\left(0, \ldots, x^{\underline{a}}, 0 \ldots\right), x_{j}^{b}=\left(0, \ldots, x^{b}, 0 \ldots\right)$ be vectors of $R^{s}$ ( $x$ at the $i^{\text {th }}$ componente, $x^{\underline{b}}$ at the $j^{i t h}$ componente). $x_{i}^{a}<x_{j}^{b}$ if either $i<j$ or $i=j$ and $x=<x^{\underline{b}}$. Furthermore we will use the notation $x_{i}^{\mathrm{u}} \mid x_{j}^{+}$iff $i=j$ and $x^{\underline{a}} \mid x^{\underline{b}}$

Using this order we can associate to $\left(g_{1}, \ldots, g_{s}\right) \in R^{s}(\neq(0, \ldots, 0))$ the leading vector and leading coefficient: Let $i$ be minimal such that $g_{i} \neq 0$ and $g_{i}=\Sigma c_{q} x$. Let $\underline{a}$ be defined by $c_{a} \neq 0$ and $c_{\mathrm{b}} \neq 0$ implies $x \underline{a} \leq x^{\underline{b}}$. Then $\mathrm{L}\left(g_{1} \ldots, g_{\mathrm{s}}\right)$ : $=x_{\mathrm{f}}^{\mathrm{q}}$ and $c\left(g_{1}, \ldots, g_{s}\right):=c_{a}$. Similar we associate to any submodule $M \subseteq R^{s}$ the leading module $L(M)$ generated by $\{L(x), x \in M, x \neq 0\}$. The leading module gives us the possibility to compute the dimension of $R^{\mathrm{s}} / M$ in a combinatorial way: $\operatorname{dim}_{c} R^{\mathrm{s}} / M=\operatorname{dim}_{c} R^{\mathrm{s}} / L(M)$, i.e. $\operatorname{dim}_{c} R^{1 /} / M$ is the cardinality of the set $\left\{x_{1}^{4}, x_{1}^{2}\right.$ is not dibvisible by $L(g)$ for all $g$ in a Gröbner base of $M$. 
The algorithm of Mora constructs for a module $M \subset R$ s generators of $L(M)$ :

Definition 3: Let $M \subseteq R^{s}$ be generated by $f_{1}, \ldots, f_{n},\left\{f_{n}, \ldots, f_{n}\right\}$ is called a Gröbner base of $M$ if $L(M)$ is generated $L(f), \ldots, L(f)$.

To describe the construction of the Gröbner base we need the following notations:

(1) Let $\left(f_{1}, \ldots, f_{\mathrm{s}}\right) \in \mathrm{R}, k\left(f_{1}, \ldots, f_{\mathrm{s}}\right):=\min \left\{i, f_{\mathrm{i}} \neq 0\right\}, k(0, \ldots, 0):=0$

(2) Let $f, g \in R^{\mathrm{s}}, k:=k(f)=k(g)>0, \mathrm{~L}(f)=x_{\mathrm{k}}^{\mathrm{b}}, \mathrm{L}(g)=x_{\mathrm{k}}^{\mathrm{b}}$, and $m=\operatorname{lcm}\left(x^{\mathrm{a}}, x^{\mathrm{b}}\right)$ then $\mathrm{S}(f, g):=\frac{m}{x^{2}} f-\frac{c(f) m}{c(g) x^{\underline{2}}} g$. This procedure associating to $f$ and $g$ the $\mathrm{S}$ vector $\mathrm{S}(f, g)$ cancels the leading terms of $f$ and $g$ and creates a new leading term if $S(f, g) \neq 0$.

(3) Let $f \in R$ be a polynomial, $f=\Sigma c_{a} x^{\text {t }}$ and let $\underline{a}^{(1)}, \underline{a}^{(2)}$ be defined by $-c_{a}^{(1)} \neq 0, c_{a}^{(2)} \neq 0$

$-c_{a} \neq 0$ implies $x^{a(i)} \leq x^{a} \leq x^{(2 / 2)}$

We define the maxmin-degree of $f$ by $d \max \min (f):=\sum\left(a_{i}^{(1)}-a_{i}^{(2)}\right)$. Let $f=\left(f_{1}, \ldots, f_{s}\right) \in R^{s}$ and $k:=k(f)>0$ then $d \operatorname{maxmin}(f):=d \operatorname{maxmin}\left(f_{k}\right)$, $d \operatorname{maxmin}(0):=0$.

The algorithm to construct the Gröbner base uses the following algorithm to decide whether a given vector is in the submodule or not:

Let $S \subseteq R^{\text {s }}$ be a finite set of vectors of polynomials, $0 \neq h \in \mathrm{R}^{\mathrm{s}}$ a vector of polynomials and $M$ the submodule of $R^{s}$ generated by $S$.

$1^{\text {st }}$ step

Let $T_{1}:=S$ and $h_{1}:=h$

$\mathrm{i}^{\text {th }}$ step

Suppose $T_{\mathrm{i}-1}$ and $h_{\mathrm{i}-\mathrm{i}}$ are defined and $h_{\mathrm{i}-1} \neq 0$. Suppose $L\left(h_{\mathrm{i}-1}\right)$ is divisible by $L(g)$ for some $g \in T_{i-1}$ then we choose $g \in T_{i-1}$ with the following properties:

(1) $\mathrm{L}(g) \mid \mathrm{L}\left(h_{\mathrm{i}-1}\right)$

(notice that this implies $k(g)=k\left(h_{\mathrm{j}-1}\right)$ by definition!)

(2) $\max \left(d \operatorname{maxmin}(g), d \operatorname{maxmin}\left(h_{\mathrm{j}-1}\right)\right)$ is minimal between all possible choices of $g$ with property (1).

We define $h_{\mathrm{i}}:=S\left(h_{\mathrm{i}-1}, g\right)$ and $T_{\mathrm{i}}:=T_{\mathrm{i}-1} \cup\left\{h_{\mathrm{i}-1}\right\}$. If $\mathrm{L}\left(h_{\mathrm{i}-1}\right)$ is not divisible by $\mathrm{L}(g)$ for all $g \in T_{\mathrm{i}-1}$ then $h \notin M$. 
Mora proved (cf. [6]) that there is an $l$ such either $h_{i}=0$ or $\mathrm{L}\left(h_{l}\right)$ is not divisible by $\mathrm{L}(g)$ for all $g \in T_{t-1}$.

We define reduction ${ }_{s}(h):=h_{i}$.

\section{The algorithm:}

Let $M \subseteq R^{s}$ be a submodule generated by $f_{1}, \ldots, f_{n} ; f_{1}$ vectors of polynomials.

We construct the Gröbner base inductively:

\section{1 st step}

We defined $S_{1}:=\left\{f_{1}, \ldots f_{\mathrm{n}}\right\}, \mathrm{P}_{1}:=\left\{\left(f_{i} f_{j}\right), i<j\right\}$.

\section{$\mathrm{j}^{\text {th }}$ step}

Suppose $\mathrm{S}_{\mathrm{i}-1}$ and $P_{\mathrm{i}-1}$ are defined.

If $P_{\mathrm{i}-1}=\varnothing$ then $S_{\mathrm{i}-1}$ is a Gröbner base of $M$.

If $P_{\mathrm{i}-1} \neq \varnothing$ we choose $(f, g) \in P_{\mathrm{i}-1}$.

Let $s=S(f, g)$ and $h=$ reduction $_{s_{1-1}}(s)$.

If $h=0$ then $S_{:}:=S_{i-1}$ and $P_{i}=P_{i-1}^{-1} \backslash\{(f, g)\}$.

If $h \neq 0$ then $S_{\mathrm{i}}:=S_{\mathrm{i}-1} \cup\{h\}$ and $P_{\mathrm{i}}:=\left(P_{\mathrm{i}-1} \backslash\{(f, g)\}\right) \cup\left\{(v, h), v \in S_{\mathrm{i}-1}\right\}$.

Now $L(M)$ is finitly generated. This implies that for some $N$ always reduction $_{S_{\mathrm{N}}}(s)=0$, i.e. the algorithm will stop and $S_{\mathrm{N}}$ is a Gröbner base.

\section{SINGULARITIES WITH EXACT POINCARE COMPLEX BUT NOT QUASIHOMOGENEOUS}

Let $(X, 0)$ be the germ of the curve singularity in $\left(C^{3}, 0\right)$ defined by the zero set of the polynomials $f=x y+z^{i-1}$ and $g=x z+y^{k-1}+y z^{2}, 4 \leq l \leq k$ and $5 \leq k$. This singularity is denoted by $\mathrm{FT}_{k, l}$ in C.T.C. Walls classification and has Milnor number $\mu(X, 0)=k+l+2$ (cf. [11]). This singularity is not quasihomogeneous. To prove this we have to compute the Tjurina number $\tau(X, 0)$ $=\operatorname{dim}_{C} C\{x, y, z\} /,\left(f, g, M_{1}, M_{2}, M_{3}\right), M_{i}$ being the 2-minors of the Jacobian matrix

$$
J(f, g)=\left(\begin{array}{ccc}
y & x & (l-1) z^{L-2} \\
z & z^{2}+(k-1) y^{k-2} & x+2 y z
\end{array}\right)
$$

$M_{1}=x z-y z^{2}-(k-1) y^{k-1}$

$M_{2}=x y+2 y^{2} z-(l-1) z^{i-1}$

$M_{3}=x^{2}+2 x y z-(l-1) z^{l}-(l-1)(k-1) y^{k-2} z^{i-2}$ 
We use Mora's algorithm to compute a Gröbner base of $\left(f, g, M_{1}, M_{2}, M_{3}\right)$ : $h_{1}:=S\left(g, M_{1}\right)=2 y z^{2}+k y^{k-1}$

$h_{2}:=S\left(M_{2} f\right)=2 y^{2} z-l z^{i-1}$

$h_{3}:=S\left(h_{1}, h_{2}\right)=l z^{i-1}+\mathrm{ky}^{k-1}$

$h_{4}:=$ reduction $_{\left[f, g, \mathrm{M}_{1}, \mathrm{M}_{2}, \mathrm{M}_{3}\right.}(\mathrm{S}(f, g))=((4-(2-l)(2-\mathrm{k})) / 2 l) y^{k}$

Let $S:=\left\{f, g, M_{3}, h_{1}, h_{2}, h_{3}, h_{4}\right\}$ then one can check that reduction ${ }_{S}(S(h, k))=0$ for all $h, k \in S$, i.e. $S$ is a Grobner base of the ideal $\left(f . g, M_{1}, M_{2}, M_{3}\right)$. This implies

$$
\begin{aligned}
\tau(X, 0) & =\operatorname{dim}_{C} C\{x, y, z\} /\left(f, g, M_{1}, M_{2}, M_{3}\right) \\
& =\operatorname{dim}_{C} C\{x, y, z\} /\left(x y, x z, x^{2}, y z^{2}, y^{2} z, z^{i-1}, y^{k}\right) \\
& =l+k+1 \\
& <l+k+2=\mu(X, 0),
\end{aligned}
$$

i.e. $(X, 0)$ is not quasihomogeneous.

We will now prove that the Poincare complex of $(X, 0)$ is exact. We have to prove (cf. introduction) that $\mu(X, 0)=\operatorname{dim} \Omega_{\mathrm{x}, 0}^{2}-\operatorname{dim} \Omega_{\mathrm{x}, 0}^{3}$

$\Omega_{\mathrm{x}, 0}^{3}=C\{x, y, z\} /,\left(\frac{\partial f}{\partial x}, \frac{\partial f}{\partial y}, \frac{\partial f}{\partial z}, \frac{\partial g}{\partial x}, \frac{\partial g}{\partial y}, \frac{\partial g}{\partial z}\right)$

$=C\{x, y, z\} /(x, y, z)$

i.e. $\operatorname{dim} \Omega_{x, 0}^{3}=1$.

We have to prove that $\operatorname{dim} \Omega_{\mathrm{X}, 0}^{2}=l+k+3$.

$\left.\Omega_{\mathrm{x}, 0}^{2}=C\left\{x_{1}, z_{2}\right\}_{2}\right\}^{3} M$ and $M$ is generated by

$f_{1}:=(f, 0,0)=\left(x y+z^{i-1}, 0,0\right)$

$f_{2}:=(0, f, 0)=\left(0, x y+z^{\prime-1}, 0\right)$

$f_{3}:=(0,0, f)=\left(0,0, x y+z^{l-1}\right)$

$f_{4}:=(g, 0,0)=\left(x z+y z^{2}+y^{k-1}, 0,0\right)$

$f_{5:}=(0, g, 0)=\left(0, x z+y z^{2}+y^{k-1}, 0\right)$

$f_{6}:=(0,0, g)=\left(0,0, x z+y z^{2}+y^{k-1}\right)$

$f_{r}:=\left(\frac{\partial f}{\partial y}, \frac{\partial f}{\partial z}, 0\right)=\left(x,(l-1) z^{l-2}, 0\right)$.

$f_{8}:=\left(\frac{\partial f}{\partial x}, 0,-\frac{\partial f}{\partial z}\right)=\left(y, 0,-(l-1) z^{l-z}\right)$

$f_{s}:=\left(0, \frac{\partial f}{\partial x}, \frac{\partial f}{\partial y}\right)=(0, y, x)$

$f_{10}=\left(\frac{\partial g}{\partial y}, \frac{\partial g}{\partial z}, 0\right)=\left(z^{2}+(\mathrm{k}-1) y^{k-2}, x+2 y z, 0\right)$

$f_{i r}:=\left(\frac{\partial g}{\partial x}, 0,-\frac{\partial g}{\partial z}\right)=(z, 0,-x-2 y z)$

$f_{12}:=\left(0, \frac{\partial g}{\partial x}, \frac{\partial g}{\partial y}\right)=\left(0, z, z^{2}+(k-1) y^{k-2}\right)$ 
Let $S_{1}:=\left(f_{1}, \ldots, f_{12}\right)$.

$h_{1}:=\operatorname{reduction}_{s_{1}}\left(S\left(f_{10}, f_{11}\right)\right)=\left(0, x+2 y z, x z+2 y z^{2}+(l-1)(k-1) y^{k-2} z^{i-2}\right)$

$S_{2}:=S_{1} \cup\left\{h_{1}\right\}$

$h_{2}:=\operatorname{reduction}_{s_{2}}\left(S\left(f_{4}, f_{11}\right)\right)=\left(0,0, x^{2}+2 x y z+(l-1) z^{i+1}+(l-1) y^{k-2} z^{l-1}\right)$

$S_{3}:=S_{2} \cup\left\{h_{2}\right\}$

$h_{3}:=$ reduction $_{s_{3}}\left(S\left(f_{6}, f_{3}\right)\right)=\left(0,0,(l / 2-1) z^{l}+y^{*}\right)$

$S_{4}:=S_{3} \cup\left\{h_{3}\right\}$

$h_{4}:=$ reduction $_{S_{4}}\left(S\left(f_{8}, f_{11}\right)\right)=\left(0,0,2 y^{2} z-l z^{\prime-1}\right)$

$S_{5}:=S_{4} \cup\left\{h_{4}\right\}$

$h_{s}:=$ reduction $_{s_{5}}\left(S\left(f_{s}, f_{12}\right)\right)=\left(0,0,-2 y z^{2}-k y^{k-1}\right)$

$S_{6}:=S_{3} \cup\left\{h_{3}\right\}$

$h_{6}:=$ reduction $_{\mathrm{s}_{6}}\left(S\left(h_{4}, h_{5}\right)\right)=\left(0,0,((2 l) /(l-2)-k) y^{*}\right)$

$S_{7}:=S_{6} \cup\left\{h_{6}\right\}$

Now one can check that reduction $s_{7}(\mathrm{~S}(g, h))=0$ for all $g, h \in S_{7}$, i.e. $S_{7}$ is a Gröbner base of $M$. Especially $\mathrm{L}(M)$ is generated by $(x, 0,0),(y, 0,0)$, $(z, 0,0),(0, x, 0),(0, y, 0),(0, z, 0),(0,0, x y),(0,0, x z),\left(0,0, y z^{2}\right),\left(0,0, x^{2}\right),\left(0,0, y^{2} z\right)$, $\left(0,0, z^{n}\right),\left(0,0, y^{\mathrm{k}}\right)$. This implies that $\operatorname{dim} \Omega_{\mathrm{x}, 0}^{2}=l+k+3$.

Let $(X, 0)$ be the germ of the curve singularity in $\left(C^{3}, 0\right)$ defined by the zero set of the polynomials $f=x y$ and $g=x z+y z^{2}+z^{3}+z^{3+i}, i>1$. This singularity is denoted by $F Z_{0, j}$ in C.T.C. Walls classification and has Milnor number $\mu(X, 0)=10+i$ (cf. [11]). As before one compute $\tau(X, 0)=9+i, \operatorname{dim}_{c} \Omega_{\mathrm{x}, 0}^{3}=1$ and $\operatorname{dim}_{C} \Omega_{\mathrm{X}, 0}^{3}=11+i$. This implies that $(X, 0)$ is not quasihomogeneous but the Poincarè complex is exact, because $f$ and $g$ satisfy Reiffens condition $(f, g) \Omega_{C^{3}, 0} \subseteq d\left((f, g) \Omega_{C^{3}, 0}^{2}\right)$ (cf. introduction). 
Singularities with exact Poincaré Complex

\section{THE INVARIANTS OF THE UNIMODAL COMPLETE INTERSECTION SINGULARITIES}

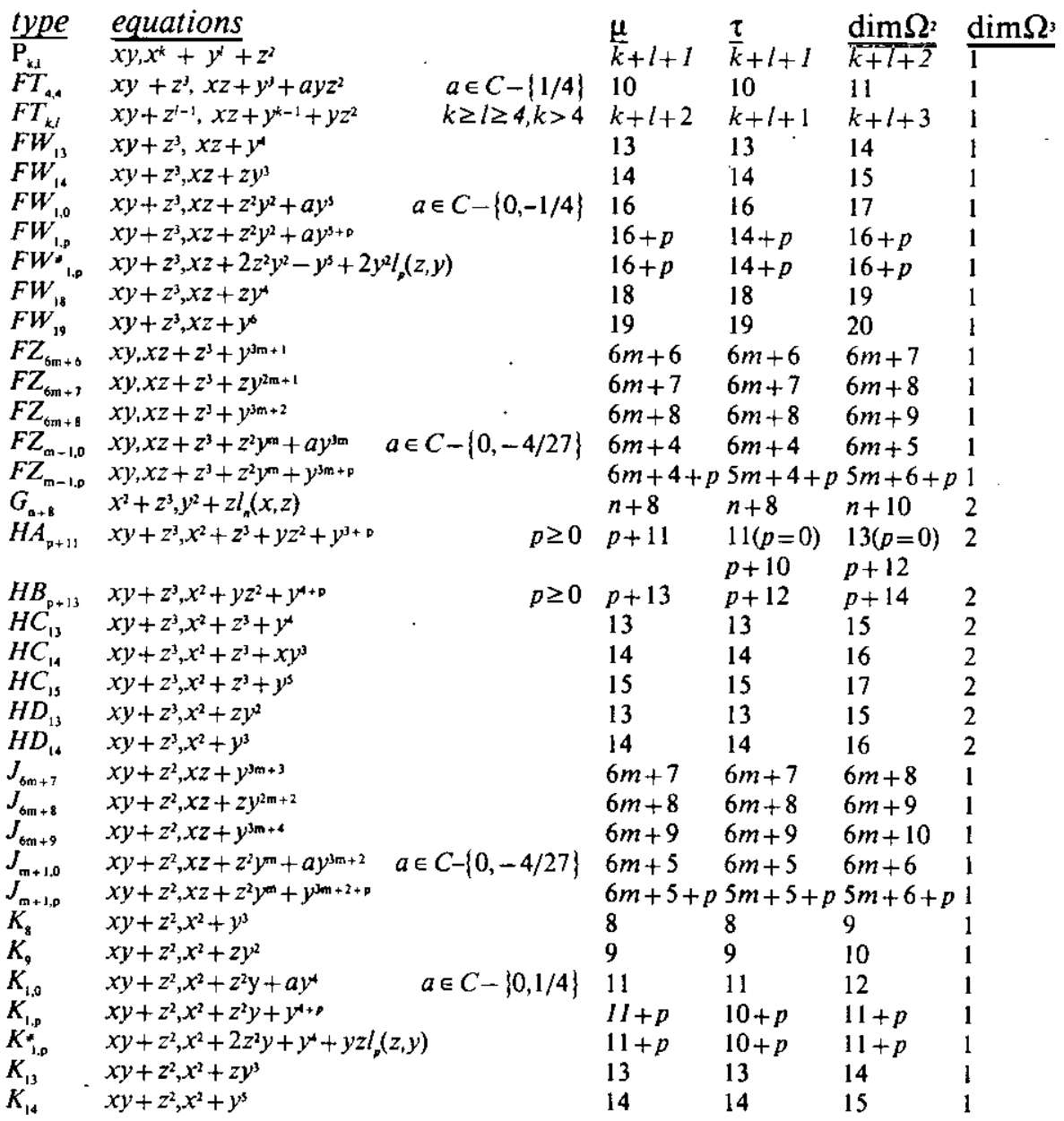

with $l_{2}(x, y)=x y^{y}$ and $l_{z+1}(x, y)=y^{* 2}$ 


\section{THE QUASIHOMOGENEOUS UNIMODAL SINGULARITIES}

Here we compare Wall's classification with the classification of Aleksandrov (cf.[1]).

\begin{tabular}{|c|c|c|}
\hline type & type & weights; degress \\
\hline$\overline{(W a} l l)$ & (Aleksandrov) & \\
\hline$P_{k, l}$ & $\begin{array}{l}l=3: T_{k+4} \\
l=4: R_{k+5} \\
l \geq 5: L\end{array}$ & $2 l, 2 k, k l ; 2(k+1), 2 k+l$ \\
\hline$F T_{4,4}$ & $V_{10}$ & $2,1,1 ; 3,3$ \\
\hline$F W_{13}^{4.4}$ & $Q_{13}$ & $11,4,5 ; 15,16$ \\
\hline$F W_{14}$ & $Q_{14}^{13}$ & $9,3,4 ; 12,13$ \\
\hline$F W_{1,0}^{14}$ & $Q_{16}$ & $7,2,3 ; 9,10$ \\
\hline$F W_{18}^{1,0}$ & & $12,3,5 ; 15,17$ \\
\hline$F W_{10}$ & & $17,4,7 ; 21,24$ \\
\hline 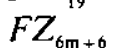 & $V_{12}\left(F Z_{12}\right)$ & $6 m+2,3,3 m+1 ; 6 m+5,9 m+3$ \\
\hline$F Z_{\text {om+o }}$ & $V_{13}\left(F Z_{3}\right)$ & $4 m+2,2,2 m+1 ; 4 m+4,6 m+3$ \\
\hline$F Z_{6 \mathrm{~m}+8}$ & $V_{14}\left(F Z_{14}\right)$ & $6 m+4,3,3 m+2 ; 6 m+7,9 m+6$ \\
\hline$F Z_{\text {m- } 1.0}$ & $V_{10}\left(F Z_{0,0}\right)$ & $2 m, 1, m ; 2 m+1,3 m$ \\
\hline$G_{n+8}$ & $M_{n+8}\left(P L_{1,0}\right)$ & $6, n+5,4 ; 12,2 n+10(n$ even $)$ \\
\hline$H A_{11}$ & $Y_{\mu}$ & $3,2,2 ; 6,5$ \\
\hline$H C_{13}$ & $L_{324}$ & $3,6,4 ; 12,9$ \\
\hline$H C_{14}$ & $G_{1,4}^{-24}$ & $9,4,6 ; 18,13$ \\
\hline$H C_{15}$ & $L_{3,5}$ & $6,15,10 ; 30,21$ \\
\hline$H D_{13}$ & $H_{13}$ & $7,5,4 ; 12,14$ \\
\hline$H D_{14}$ & $H_{14}$ & $9,6,5 ; 15,18$ \\
\hline$J_{6 \mathrm{~m}+7}$ & $U_{13}\left(J_{13}\right)$ & $6 m+5,3,3 m+4 ; 6 m+8,9 m+9$ \\
\hline$J_{b+i+i}$ & $U_{14}\left(J_{14}\right)$ & $4 m+4,2,2 m+3 ; 4 m+6,6 m+7$ \\
\hline$J_{6 m+9}$ & $U_{15}\left(J_{13}\right)$ & $6 m+7,3,3 m+5 ; 6 m+10,9 m+12$ \\
\hline$J_{\mathrm{m}+1.0}$ & $U_{11}\left(J_{2,0}\right)$ & $2 m+1,1, m+1 ; 2 m+2,3 m+2$ \\
\hline$K_{8}+1,0$ & $Y_{8}$ & $6,4,5 ; 10,15$ \\
\hline$K_{0}$ & $M_{0}$ & $5,3,4,8,10$ \\
\hline$K_{1,0}$ & $M_{11}$ & $4,2,3 ; 6,8$ \\
\hline$K_{13}$ & $N_{13}$ & $7,3,5 ; 10,14$ \\
\hline$K_{14}$ & $N_{14}$ & $10,4,7 ; 14,20$ \\
\hline
\end{tabular}




\section{References}

[1] AlEksandrov, A.G. Normal forms of one dimensional quasihomogeneous complete intersection singularities. Math. Sbornik 117 (159):1, 3-31 (1982).

[2] BUCHBERGER, B. An Algorithm for Finding a Basis for the Residue Class Ring of a Zero-dimensional Polynomial Ideal. Ph. D. Thesis, Univ. of Innsbruck (Austria), Dep. of Math., 1965.

[3] GREUEL, G.M. On deformation of curves and a formula of Deligne. Lecture Notes 901, 141-168.

[4] GReuel, G.M. Dualität in der lokalen Kohomologie isolierter Singularitaten. Math. Ann. 250, 157-173 (1980).

[5] Greuel, G.M.; Martin; B.; Pfister, G. Numerische Charakterisierung quasihomogener Gorenstein-Kurvensingularitäten. Math. Nachr. 124 (1985), 123-131.

[6] MORA, F. A constructive characterisation of standard bases. Boll. U.M.I. sez. D, 2(1983), 41-50.

[7] REIFFEN, H.J. Kontrahierbare analytische Mengen. Math. Z. 109 (1969) 253-268.

[8] SAITO K. Quasihomogene isolierte Singularitäten von hyperflächen. Inv. math. 14 (1971) 123-142.

[9] WAHL, J. A characterization of quasihomogeneous Gorenstein surface singularities. Composit. Math. 55 (1985) 269-288.

[10] WALDI, R. Deformationen von Gorenstein-Singularitäten der Kodimension 3. Math. Ann. 242 (1979) 201-208.

[11] WALL, C.T.C. Classification of unimodal isolated singularities of complete intersections. Proc. Symp. Pure Math. 40, 2(1983) 625-640.

Humboldt-Universität zu Berlin

Sektion Mathematik

PF 1297

1080 Berlin

DDR 